\title{
Experimental Study of Showerhead Cooling on a Cylinder Comparing Several Configurations using Cylindrical and Shaped Holes
}

\author{
H. Reiss, A. Bölcs \\ Swiss Federal Institute of Technology \\ Lausanne, Switzerland
}

\begin{abstract}
Film cooling and heat transfer measurements on a cylinder model have been conducted using the transient thermochromic liquid crystal technique. Three showerhead cooling configurations adapted to leading edge film cooling of gas turbine blades were directly compared: 'classical' cylindrical holes versus two types of shaped hole exits. The experiments were carried out in a free jet test facility at two different flow conditions, Mach numbers $M=0.14$ and $M=0.26$, yielding Reynolds numbers based on the cylinder diameter of $8.6 \mathrm{e} 4$ and $1.55 \mathrm{e}$, respectively. All experiments were done at a main stream turbulence level of $\mathrm{Tu}=7 \%$ with an integral lengthscale of $\mathrm{L}_{\mathrm{x}}=9.1 \mathrm{~mm}$ $(\mathrm{M}=0.14)$, or $\mathrm{L}_{\mathrm{x}}=10.5 \mathrm{~mm} \quad(\mathrm{M}=0.26)$ respectively. Foreign gas injection $\left(\mathrm{CO}_{2}\right)$ was used yielding an engine-near density ratio of 1.6, with blowing ratios ranging from 0.6 to 1.5 .

Detailed experimental results are shown, including surface distribution of film cooling effectiveness and local heat transfer coefficients. Additionally, heat transfer and heat load augmentation due to injection with respect to the uncooled cylinder are reported.

For a given cooling gas consumption the laid-back shaped hole exits lead to a clear enhancement of the cooling performance compared to cylindrical exits, whereas laterally expanded holes give only slight performance enhancement.
\end{abstract}

\begin{tabular}{lll}
\multicolumn{3}{l}{ NOMENCLATURE } \\
$\mathrm{A}$ & {$\left[\mathrm{m}^{2}\right]$} & surface area \\
$\mathrm{C} \mathrm{p}$ & {$[\mathrm{J} / \mathrm{kgK}]$} & specific heat at constant pressure \\
$\mathrm{d}$ & {$[\mathrm{mm}]$} & leading edge, diameter, hole diameter \\
$\mathrm{D}$ & {$[\mathrm{mm}]$} & cylinder diameter \\
$\mathrm{DR}$ & {$[-]$} & coolant-to-gas density ratio $\rho_{\mathrm{c}} / \rho_{\mathrm{g}}$ \\
$\mathrm{G}$ & {$[-]$} & bulk blowing ratio $\mathrm{u}_{\mathrm{c}} \rho_{\mathrm{c}} / \mathrm{u}_{\mathrm{g}} \rho_{\mathrm{g}}$ \\
$\mathrm{I}$ & {$[-]$} & bulk momentum flux ratio $\mathrm{u}_{\mathrm{c}}{ }^{2} \rho_{\mathrm{c}} / \mathrm{u}_{\mathrm{g}}{ }^{2} \rho_{\mathrm{g}}$ \\
$\mathrm{L}$ & {$[\mathrm{m} / \mathrm{s}]$} & cooling hole length \\
$\mathrm{L}_{\mathrm{x}}$ & {$[\mathrm{m}]$} & longitudinal integral lengthscale \\
$\mathrm{M}$ & {$[-]$} & Mach number $\mathrm{u} /(\mathrm{kRT})$ \\
$\mathrm{Nu}$ & {$[-.5$} \\
$\mathrm{n}$ & {$[-]$} & Nusselt number $\alpha \mathrm{D} / \lambda$ \\
$\mathrm{p}$ & {$[\mathrm{Pa}]$} & summation index \\
$\mathrm{Q}$ & {$[\mathrm{W}]$} & pressure \\
$\mathrm{q}$ & {$\left[\mathrm{W} / \mathrm{m}^{2}\right]$} & speat flux \\
$\mathrm{R}$ & {$[\mathrm{J} / \mathrm{kg} / \mathrm{K}]$} & ideal gas constant \\
$\mathrm{Re} \mathrm{D}_{\mathrm{D}}$ & {$[-]$} & Reynolds number $(\mathrm{uD}) / \mathrm{v}$ \\
$\mathrm{R}_{\mathrm{a}}, \mathrm{R}_{\mathrm{z}}, \mathrm{R}_{\mathrm{t}}[\mu \mathrm{m}]$ & surface roughness parameters (DIN4768) \\
$\mathrm{r}_{0}$ & {$[-]$} & recovery factor \\
$\mathrm{T}$ & {$[\mathrm{K}]$} & temperature \\
$\mathrm{t}$ & {$[\mathrm{s}]$} & time \\
$\mathrm{Tu}$ & {$[\%]$} & turbulence intensity \\
$\mathrm{u}$ & {$[\mathrm{m} / \mathrm{s}]$} & velocity \\
& &
\end{tabular}

\begin{tabular}{|c|c|c|}
\hline & {$[\mathrm{m}]$} & model depth \\
\hline \multicolumn{3}{|c|}{ GREEK } \\
\hline$\alpha$ & {$\left[\mathrm{W} /\left(\mathrm{m}^{2}\right.\right.$} & K)]local heat transfer coefficient \\
\hline$\beta$ & {$\left[{ }^{\circ}\right]$} & spanwise inclination angle \\
\hline$\gamma$ & {$\left[{ }^{\circ}\right]$} & surface angle, exit location angle \\
\hline$\eta$ & [- ] & film cooling effectiveness \\
\hline$\varphi$ & {$\left[{ }^{\circ}\right]$} & streamwise inclination angle \\
\hline$\rho$ & {$\left[\mathrm{kg} / \mathrm{m}^{3}\right]$} & density \\
\hline$\tau$ & {$[-]$} & summation index \\
\hline$\mu$ & {$\left[\mathrm{Ns} / \mathrm{m}^{2}\right]$} & dynamic viscosity \\
\hline$v$ & {$\left[\mathrm{~m}^{2} / \mathrm{s}\right]$} & kinematic viscosity \\
\hline$\kappa$ & {$[-]$} & isentropic coefficient $\mathrm{c}_{\mathrm{p}} / \mathrm{c}_{\mathrm{V}}$ \\
\hline$\lambda$ & {$[\mathrm{J} / \mathrm{mK}]$} & thermal conductivity \\
\hline$\Lambda$ & {$\left[\mathrm{m}^{2} / \mathrm{s}\right]$} & thermal diffusivity $\lambda /\left(\rho c_{p}\right)$ \\
\hline$\Phi$ & {$[-]$} & overall effectiveness \\
\hline \multicolumn{3}{|c|}{ SUBSCRIPTS } \\
\hline av & & adiabatic wall \\
\hline c & & coolant \\
\hline f & & film cooling \\
\hline g & & main stream gas \\
\hline i & & initial \\
\hline $\mathrm{r}$ & & recovery \\
\hline $\mathrm{s}$ & & static conditions, surface \\
\hline $\mathrm{t}$ & & total conditions \\
\hline 0 & & reference, cooling hole base \\
\hline
\end{tabular}

\section{INTRODUCTION}

The further enhancement of the specific power output and thermal efficiency of modern gas turbines goes along with continuously increasing turbine inlet temperatures over recent years. Due to operation temperatures that largely exceed the allowable material temperatures, extensive cooling of the first stage(s) of the turbine is necessary in order to ensure high reliability of the 'hot' components and to achieve long life cycles of the blading. Currently, internal cooling via convection and impingement is combined with extensive external cooling via film cooling. For the correct thermal design of turbine components, detailed knowledge of heat transfer rates and metal temperatures are required. Today, however, high uncertainties for the local heat transfer persist, yielding even higher uncertainties for the local material temperatures. Therefore, it is yet difficult to determine the optimum thermal design. This situation can be improved by providing accurate heat transfer data - gathered with numerical tools, or experimentally - for realistic, 'near-engine' cooling situations. 
One of the focal points of cooling research is the optimization of film cooling schemes, aimed at maximizing the external cooling effects with a minimum coolant gas consumption. This can be achieved for example by finding the optimum configuration of injection holes. In this context, the use of sophisticated hole arrangements with 'shaped' exits - rather than simple cylindrical holes - has to be mentioned as a very promising option. In fact, considerable improvement of cooling performance has been reported by several researchers for simplified situations.

\section{Previous Work on Shaped Holes}

Several experimental studies on flat plate film cooling with shaped holes can be found in the literature, generally indicating a positive effect of shaped holes on the cooling performance.

Goldstein et al. (1973) carried out measurements on a flat plate equipped with a single cooling hole, and compared a cylindrical hole exit to a conically widened one. Increased cooling effectiveness was detected for widened hole exits both in downstream and lateral directions. It was reasoned that the reduced momentum of the jet due to hole expansion towards the exit reduces the jet's tendency to detach from the model surface, thus reducing penetration of the coolant into the boundary layer. As a result, less mixing and better coverage occur.

Wittig et al. (1996)) tested a single cooling hole with inclination in the flow direction on a large-scale model at trans- and supersonic flow conditions. A cylindrical hole was compared with two kinds of shaped holes - laterally expanded, and laid back \& laterally expanded. It was reported that the tendency of jet lift-off is lowered by inclining injection holes into flow direction, or shaping hole exits to obtain 'smoother' injection. Also here, this was attributed to a reduced momentum of the injected secondary fluid due to a diffuser effect of the widened exits.

Other studies investigated an entire cooling row instead of individual holes, also indicating positive effect of diffuser-shaped exits Beeck et al. (1993); Giebert et al. (1997); Bittlinger et al. (1994).

These studies have been done on various model geometries and cooling configurations. However, a direct quantitative comparison between the different hole shapes is difficult since often the configurations differ not only in exit shape, but also other geometrical parameters (number of injection rows, hole spacing, orientation, exit shape etc.). Moreover, a mismatch of other experimental parameters such as flow conditions may make direct comparisons impossible. For high curvature situations such as on the leading edge, or the pressure or suction sides of turbine blades, not much data on shaped holes, in combination with several rows of injection holes is published to the knowledge of the authors. It is therefore hard to conclude from the literature data on the actual benefit of shaped holes for leading edge cooling.

\section{Present Study}

The objective of the present study is to explore the potential improvement of film cooling with shaped holes for the leading edge region. In particular it is of interest to investigate the combined effect of high surface curvature, stagnation point flow with a very thin boundary layer and high flow acceleration, and the interaction of several rows of cooling holes as they are currently found in gas turbines. Another aim of this study is to provide an experimental data set allowing the direct comparison of several injection schemes for a complex and realistic situation, which might be useful for both gas turbine design and code validation.

\section{EXPERIMENTAL SETUP}

\section{Free Jet Test Facility}

The experiments were carried out in a free jet test facility, using a rapid exposure mechanism for a cylinder model which has been used in a previous study by Hoffs et al. (1997)). The cylinder model can be pivoted into the free jet flow as shown in Fig. 1. It is mounted on a base plate with micrometrical angle adjustment allowing for precise orientation of the showerhead with respect to the main flow. A turbulence grid is attached to the free jet exit, creating near-engine turbulence intensity values at the cylinder leading edge of about $\mathrm{Tu}=7 \%$, with a non-dimensional integral length scale of $\mathrm{L}_{\mathrm{x}} / \mathrm{D}=0.30 \%$ $(\mathrm{M}=0.14)$ or $\mathrm{L}_{\mathrm{x}} / \mathrm{D}=0.35 \quad(\mathrm{M}=0.26)$. Turbulence quantities were measured with hot-wire anemometry. The free jet exit has a diameter of 5 cylinder diameters D. The exchangeable showerhead section has a length of 2D. Once in measurement position, the cylinder leading edge is located at a distance of 5D downstream of the turbulence grid. The preconditioned coolant gas is supplied to the plenum chamber of the test section via insulated tubings. $\mathrm{CO}_{2}$ is used as coolant gas, yielding a density ratio of $\mathrm{DR} \approx 1.6$. The model surface is viewed with a miniature CCD camera situated next to the free jet exit. Halogen light sources and fiber optics are used for illumination. The flow around the cylinder test section is essentially two-dimensional, which was verified with hot wire and aerodynamic probe measurements.

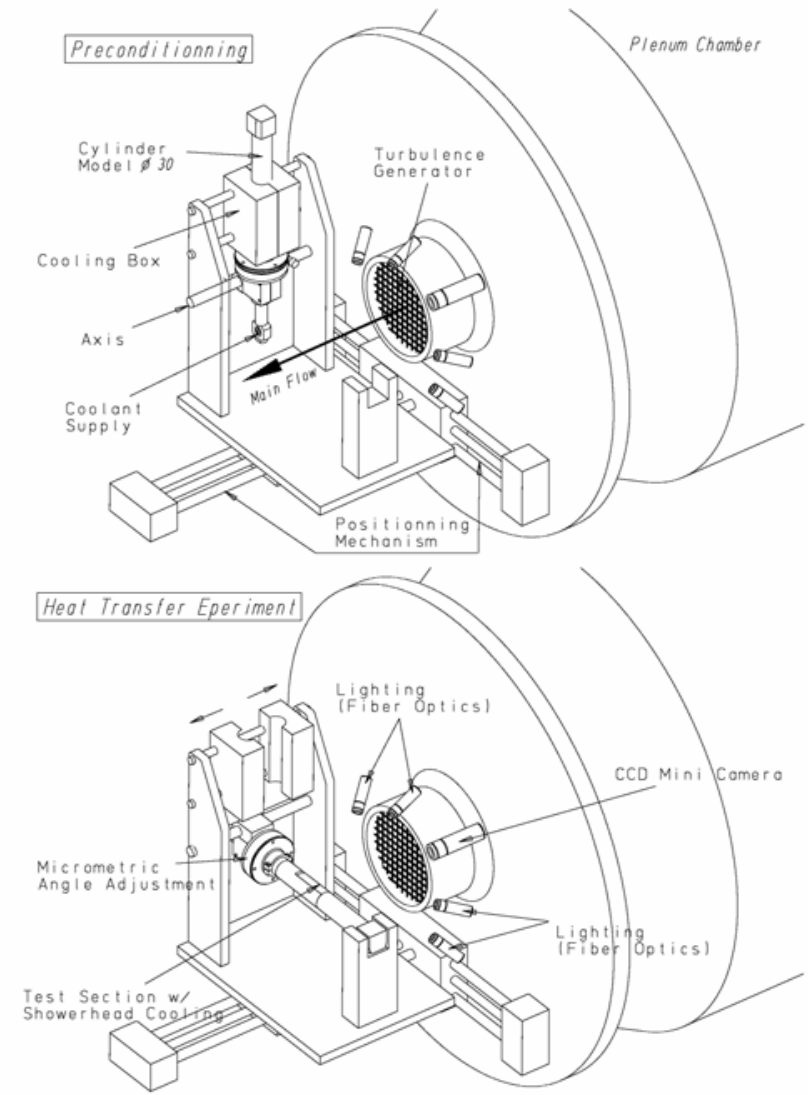

\section{Fig. 1 - Free Jet Test Facility with Pivot Mechanism for Cylinder Model}

\section{Instrumentation}

The cylinder carrying the test specimen is depicted in Fig. 2. A series of pressure taps are arranged in the circumferential direction, adjacent to the showerhead insert. To measure the initial temperature to which the model is pre-conditioned, 6 thermocouples are embedded in the Perspex test section at various locations. The cut-out on the right hand side of Fig. 2 shows the orientation of the showerhead in the main flow (thin radial lines indicate positions of pressure taps) The model surface is coated with a thin layer of commercial narrow-band thermochromic liquid crystals. These are sprayed-on prior to each 
series of experiments, and subsequently polished for repeatable and smooth surface quality. Typically, a surface roughness of $\mathrm{R}_{\mathrm{z}}=9 \pm 1.5$ $\mu \mathrm{m}, \mathrm{R}_{\mathrm{a}}=2.4 \pm 0.5 \mu \mathrm{m}$, and $\mathrm{R}_{\mathrm{t}}=15 \pm 2.7 \mu \mathrm{m}$ are achieved.

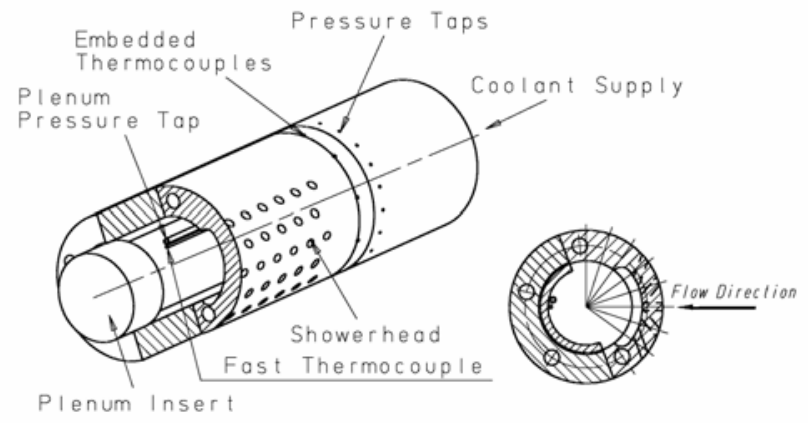

Fig. 2 - Cylinder Model Instrumentation

\section{Data Analysis}

The local heat flux onto a film-cooled surface can be written as

$$
q=\alpha_{f}\left(T_{a w}-T_{w}\right)
$$

where the driving temperature difference for the definition of $\alpha_{\mathrm{f}}$ is the adiabatic wall temperature $T_{a w}$ (which is the effective gas temperature at the wall) minus the surface temperature of the model. $T_{a w}$ is unknown and depends on the temperatures of the main stream and the injected coolant gas, and on the mixing between jets and main flow. It can be written in dimensionless form as the film cooling effectiveness

$$
\eta=\frac{T_{a w}-T_{r g}}{T_{t c}-T_{t g}}
$$

expressing how closely $\mathrm{T}_{\mathrm{aw}}$ approaches the coolant injection temperature. Both unknowns $\alpha_{f}$ and $\eta$ are functions of the aerodynamic flow field alone and do not depend on the actual choice of the temperature values, as long as constant gas properties are supposed Vedula and Metzger (1991).

The data analysis is based on the theory of one-dimensional transient heat conduction into a semi-infinite solid. The governing differential equation for the temperature evolution of the solid is

$$
\frac{\partial^{2} T}{\partial x^{2}}=\frac{1}{\Lambda} \frac{\partial T}{\partial t}
$$

with the initial condition $\left.T(x, t)\right|_{t=0}=T_{i}$. It is supposed that, during an experiment, a heat pulse enters only a short distance into the model compared to its wall thickness, i.e. the model interior remains at initial temperature $T_{i}$ at all times, and the temperature gradient $\partial T / \partial x$ is zero. This is formally expressed as the boundary condition for Eq. (3) as $\lim _{x \rightarrow \infty} T(x, t)=T_{i}$. At the surface a convective boundary condition is imposed :

$$
-\left.\lambda \frac{\partial T(x, t)}{\partial x}\right|_{x=0}=\left.\alpha_{f}\left(T_{a w}-T(x, t)\right)\right|_{x=0}
$$

Eq. (4) represents an instantaneous step change of the effective fluid temperature along the model surface from $T_{i}$ to $T_{a w}$. In reality, a true step change for the main stream temperature can closely be approximated by rapid exposure of the preconditioned model to the flow Hoffs et al. (1997). However, this is not the case for $\mathrm{T}_{\mathrm{aw}}$ since the coolant injection temperature varies gradually during a transient experiment due to internal heat exchange which occurs in the supply tubings, the plenum and particularly in the cooling holes inside of the pre-conditioned model. Thus, the adiabatic wall temperature becomes a function of time. This is accounted for by approximating the measured coolant injection temperature with a power series of typically $4^{\text {th }}$ to $5^{\text {th }}$ order as

$$
T_{t c}(t)=\sum_{n=0}^{N} A_{n} \frac{t^{n}}{\Gamma(n+1)}
$$

Employing this transient coolant temperature, the Laplace transform method yields an analytical solution for the temperature evolution at $\mathrm{x}=0$ as previously described by Drost et al. (1997)

$$
\begin{aligned}
& T_{w}-T_{i}=\left(T_{r g}-\eta T_{t g}-T_{i}\right)\left[1-e^{\beta^{2}} \operatorname{erfc}(\beta)\right]- \\
& \eta \sum_{n=0}^{N}\left\{A_{n}\left(\frac{\kappa}{\alpha_{f}}\right)^{2 n}\left[e^{\beta^{2}} \operatorname{erfc}(\beta)-\sum_{\tau=0}^{2 n}\left((-2 \beta)^{\tau} i^{\tau} \operatorname{erfc}(0)\right)\right]\right\} \\
& \text { with } \quad \kappa=\frac{\lambda}{\sqrt{\Lambda}}=\sqrt{\rho \lambda c_{p}} \text { and } \quad \beta=\frac{\alpha_{f} \sqrt{t}}{\kappa}
\end{aligned}
$$

Eq. (5) contains the two unknowns $\alpha_{f}$ and $\eta$. In order to solve for $\alpha_{f}$ and $\eta$, a multiple-regression analysis is applied: a number of 6 to 8 transient experiments is conducted at identical aerodynamic and thermal conditions but with varying the coolant temperature. The actual coolant temperature evolution at the hole exit, which is calculated based on measurements of the coolant total conditions in the plenum chamber, is shown on the left-hand side of Fig. 3. The corresponding (calculated) wall temperature rise for a given surface position is indicated on the right-hand side of Fig. 3. Since a single layer of narrow band liquid crystals is used, one "event" can be detected per chosen hue value and test, that is when the surface point passes the corresponding liquid crystal temperature $\mathrm{T}_{\mathrm{LC}}$. A leastsquare fit of Eq. (5) is applied to the ensemble of points $\left(t, T_{w}=T_{L C}\right)$ yielding both unknowns $\alpha_{\mathrm{f}}$ and $\eta$.
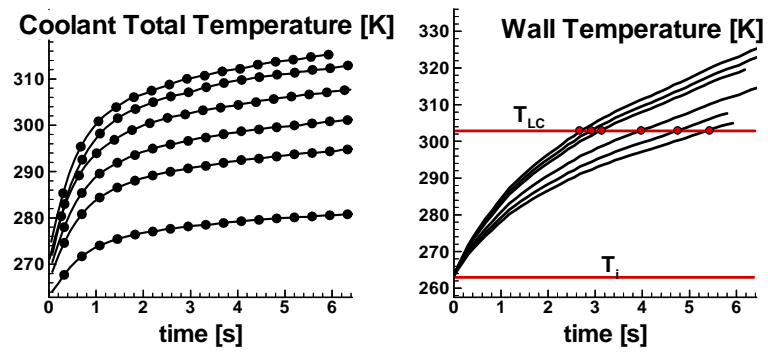

Fig. 3 - Variation of Coolant and Wall Temperatures for a Test Ensemble

This 'overdetermined' approach results in smaller uncertainties, compared to those obtained using the minimum of only two experiments. Taking into account the existing measurement uncertainties the error on the heat transfer coefficient is about $6 \%$, and on the film cooling effectiveness $4 \%$ (for $\eta=0.3$ ) to $10 \%$ (for $\eta=0.1$ ). Varying the injection temperature over a range of $30 \mathrm{~K}$ causes variations of the density ratio and blowing ratio, and momentum ratio of the order of $8 \%$ over an ensemble of tests. 


\section{Image Processing}

The color play of the liquid crystals is captured with a miniature CCD camera, that views the upper side of the test section, covering surface angles $\gamma$ from roughly $-10^{\circ}$ to $90^{\circ}$. The RGB image signal is analyzed with a special image processing computer based on the hue capturing technique which consists of a real-time conversion of the RGB signal into a hue-saturation-intensity signal and then filtering out all but a specified range of hue values which yields a considerable reduction of the data. Typically two narrow colors bands (corresponding to green and cyan) are chosen, which were calibrated individually. For the specific liquid crystals used, the temperatures of appearance of these two colors were approximately $0.5^{\circ} \mathrm{C}$ apart. The reduced image sequence is then stored and transferred to workstation for later data processing. This consists of a coordinate transformation from the image coordinates of the camera into model surface coordinates using a reference grid, followed by separating the liquid crystal events at given position and their respective time of appearance. In order to reduce the influence of the noise of the image signal on the data evaluation, a number of pixels are treated together, subdividing the model surface into a regular grid with user-defined resolution. Typically, 200 x 300 cells were used, yielding a spatial resolution of roughly 15 data points over a surface distance corresponding to one hole diameter $\mathrm{d}$.

The color events are detected using an averaging procedure in both time and space: a hue signal needs to appear at a surface position as well as neighboring positions, over a number of consecutive images in order to be considered as an event.

The type of crystals used in this study showed a good accuracy of the temperature calibration the order of $\pm 0.15^{\circ} \mathrm{C}$ for a given hue value. The influence of view angle or irregular illumination which is important for wide-band liquid crystals, can be neglected.

\section{Experimental Procedure}

The test facility runs at steady-state conditions. The main stream temperature is adjusted to $60-65^{\circ} \mathrm{C}$. Prior to the heat transfer tests, the cylinder model is preconditioned to an initial temperature of about $15^{\circ} \mathrm{C}$ using a cooling box that encloses the cylinder model (see Fig. 1). The initial temperature of the test insert is monitored using several thermocouples, as illustrated in Fig. 2. The coolant temperature is set via cooling and heating devices and heat exchangers. In order to achieve the desired variation of injection temperature, it is necessary to precondition the coolant supply tubings. Thus the coolant flow is readily established prior to the test begin, up to the actual orifice to the plenum chamber where it is by-passed around the showerhead section, into the ambient air. At the actual start of a transient experiment, the cylinder is released from the cooling box and rapidly pivoted into its measuring position in the flow. An automatic valve switches on the coolant gas from the by-pass to the plenum in the cylinder. A programmable control device regulates the flow rate during the test in order to limit flow rate fluctuations which might occur during switching over from by-pass to cooling mode; additionally the actual flow rate is continuously measured with a laminar flow element. The transient total temperature in the plenum is measured with fine fast-response thermocouples residing in the plenum center, and the coolant total pressure is acquired with a pressure tap (see also Fig. 2).

Subsequently during data processing the effective coolant temperature at the exit location is determined via an isentropic calculation, based on the measured total conditions in the plenum and the static pressure at the hole exit. The latter is interpolated for from pressure tap measurements on the cylinder surface. The error on the coolant exit temperature due to internal heat exchange depends strongly on the actual coolant temperature of the respective test. A worst case estimation has been done using a numerical simulation of the transient heat exchange in the near-hole region, yielding an error of less then $0.5^{\circ} \mathrm{C}$ which is considered negligible.

\section{Test Conditions}

An overview of the test conditions that were measured in this study is given in Table 1. All measurements were carried out at the nominal angle of attack of $0^{\circ}$, i.e. the flow was symmetrical with respect to the showerhead arrangement. The temperature ratio between main stream and secondary fluid varied from $\mathrm{T}_{\mathrm{g}} / \mathrm{T}_{\mathrm{c}}=1.0$ to 1.2 , depending on the actual coolant injection temperature of the individual test. Both the blowing and momentum flux ratios are given as bulk quantities, i.e. they are averaged for the entire showerhead. Inevitable variations of the local coolant flow rate through individual cooling rows result from the circumferential pressure distribution around the cylinder. The flow splits among the different rows could not be assessed experimentally. They can be estimated based on the measured driving pressure ratio $\mathrm{p}_{\mathrm{plen}} / \mathrm{p}_{\mathrm{s}, \text { exit }}$. However, the calculation of the mass flow distribution is sensitive to slight variations of the pressure ratio. This is especially true for small blowing ratios, for which $\mathrm{p}_{\text {plen }} / \mathrm{p}_{\mathrm{s}, \mathrm{exit}}$ is very close to unity and the quantity being injected through the middle row is low. Given the existing measurement uncertainty on the pressure ratio, the following coolant distribution has to be considered as approximately: $12 \pm 5 \%$ of the total coolant mass flow were injected the $0^{\circ}$ position, $18 \pm 5 \%$ through each of the rows at $\pm 20^{\circ}$, and $26 \pm 5 \%$ at $\pm 40^{\circ}$, with slight variations depending on the blowing ratio. Potential differences between the cylindrical and hole shapes could not be resolved.

The hot gas ingestion into the showerhead through the middle row was checked for by monitoring the pressure ratio $\mathrm{p}_{\text {plen }} / \mathrm{p}_{\text {tot }}$. Additionally the temperature transients in the plenum were checked for steep temperature peaks occurring at model insertion, which are a clear indicator of any hot gas ingestion. For bulk blowing ratios $G$ greater than 0.5 , no hot gas ingestion was detected.

To allow direct comparison both $\mathrm{G}$ and I refer to the 'inner' hole surface $A_{0}$ which is the same for all three showerhead models, rather than to the actual exit surface $\mathrm{A}$.

\begin{tabular}{|c|c|c|c|c|c|}
\hline \multicolumn{4}{|c|}{ Main Stream } & \multicolumn{2}{c|}{ Film Cooling } \\
\hline $\begin{array}{c}\mathbf{M} \\
{[-]}\end{array}$ & $\begin{array}{c}\mathbf{R e}_{\mathbf{d}} \\
{[-]}\end{array}$ & $\begin{array}{c}\mathbf{T u} \\
{[\%]}\end{array}$ & $\begin{array}{c}\mathbf{L}_{\mathbf{x}} \\
{[\mathrm{mm}]}\end{array}$ & $\begin{array}{c}\mathbf{G}_{\mathbf{C O} 2} \\
{[-]}\end{array}$ & $\begin{array}{c}\mathbf{I}_{\mathbf{C O} 2} \\
{[-]}\end{array}$ \\
\hline 0.14 & $8.6 \mathrm{e} 4$ & $7 \%$ & 9.1 & 0.6 & 0.21 \\
\hline & & & & 1.0 & 0.60 \\
\hline & & & & 1.3 & 1.02 \\
\hline & & & & 1.5 & 1.35 \\
\hline \multirow{2}{*}{0.26} & \multirow{2}{*}{$1.55 \mathrm{e} 5$} & \multirow{2}{*}{$7 \%$} & \multirow{2}{*}{10.5} & 0.6 & 0.21 \\
\cline { 4 - 5 } & & & & 1.0 & 0.60 \\
\cline { 4 - 5 } & & & & 1.3 & 1.02 \\
\cline { 4 - 5 } & & & & 1.5 & 1.35 \\
\hline
\end{tabular}

Table 1 - Test Matrix for Showerhead Experiments

\section{THE SHOWERHEAD GEOMETRIES}

The geometries for this study were specially designed for the leading edge region, using some of the features of shaped holes that were already used by Gritsch et al. (1997). However, particular design constraints for film cooling of a gas turbine leading edge have to be respected, such as limited space, relatively small wall thickness and constraints in terms of possible hole orientations. Therefore, the hole types reported in literature for flat plate situations were modified and specifically adapted for the showerhead arrangement. The resulting design is a compromise between feasibility of manufacture and complexity. Fig. 4 shows the three showerhead configurations.

The 'basic' showerhead configuration (Fig. 4, left) consists of 5 staggered rows of cylindrical holes with a diameter ratio of $d / D=0.05$, They are arranged symmetrically with respect to the free jet flow, at circumferential positions $0^{\circ}, \pm 20^{\circ}$, and $\pm 40^{\circ}$, and a hole spacing of 
$\mathrm{p} / \mathrm{d}=3.7$ in the 'spanwise' direction. The holes of all five rows are oriented by $45^{\circ}$ towards the cylinder axis (i.e. perpendicular to the main flow), whereas the holes of all neighboring rows are additionally inclined by $30^{\circ}$ in the circumferential direction (i.e. towards the local main flow direction).
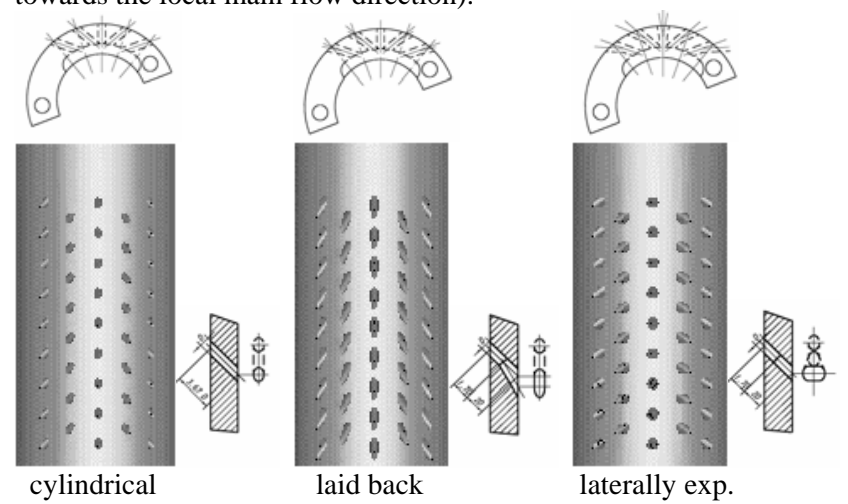

cylindrical laid back

laterally exp.

Fig. 4 - Overview over Showerhead Geometries

The 'laid back' configuration (Fig. 4, center) uses the same hole arrangement, but the exits are opened by $15^{\circ}$ in the direction of the hole inclination, over a depth of 2 hole diameters. This increased the effective surface area of the hole exits by a factor of 3.5 compared to cylindrical holes.

The third configuration (Fig. 4, right) has hole exits that are shaped laterally outwards, in a sense perpendicular to the hole inclination. This is done on both sides for the center row by an angle of $\pm 10^{\circ}$, and by $15^{\circ}$ for the neighboring rows only on one side, in the direction of main flow. The exit surface area for this configuration is increased by a factor of 1.8 with respect to the cylindrical holes.

\section{RESULTS AND DISCUSSION}

The data is presented in form of contour plots showing the surface distribution on a portion of the model, as well as in the form of spanwise averaged quantities as a function of surface angle. The contours are instructive and allow a qualitative interpretation, since they effectively illustrate the characteristics of the formation of the cooling film, in terms of the behavior of the individual jets (lift-off, re-attachment, trajectory, etc.), and the interaction between the different cooling rows. This is needed for understanding the highly 3dimensional physical effects involved in showerhead cooling. The plots contain about 160x250 data points, corresponding to a spatial resolution of approximately $1 / 15$ hole diameter d. However, in regions of high efficiencies such as in the streaks downstream of a cooling hole, the results are relatively noisy which is due to the nature of the liquid crystal signal at these points: the signal appears very fast for high coolant injection temperatures which yields high uncertainties with regards to time. In contrast, for very low injection temperatures, it may not appear at all during a transient test, i.e. the rise of the surface temperature is too slow, and the surface does not heat up to the liquid crystal temperature, yielding partly irregular spacing of the data points. This was accounted for when deriving the spanwise averaged curves by interpolating the zones of missing data. The spanwise average results are used for quantitative discussion, and meaningful comparison of the cooling performance amongst the cooling configurations.

\section{Film Cooling Effectiveness}

Detailed results of film cooling effectiveness are presented in Fig. 5 for the low Mach number case, and in Fig. 6 for the high Mach number case. Each of the figures contains 12 individual graphs, arranged in three columns for the cylindrical, laid back, and laterally expanded holes from left to right, and in four lines for the blowing ratios $G=0.6,1.0$. 1.3 and 1.5 from top to bottom. The graphs represent an unwrapped section of the model surface covering surface angles from approximately $-10^{\circ}$ to $80^{\circ}$ along the horizontal axis, and a spanwise distance corresponding to 4 axial hole spacings along the vertical axis. The cooling rows at $0^{\circ}, 20^{\circ}$ and $40^{\circ}$ can be seen from left to right ; they are subsequently referred to as row "1" (stagnation line), "2" $\left(20^{\circ}\right)$ and "3" $\left(40^{\circ}\right)$. The main flow direction is towards the right. The hole exits for the respective cooling configuration are added to the graphs (in true shape, size and position) to illustrate the actual proportions of the showerhead. The spanwise inclination of the cooling holes by $45^{\circ}$ is pointing downwards in the graphs, and the coolant supply to the plenum comes from the top. High efficiencies are indicated as black, and low as white. The corresponding spanwise averaged film cooling effectiveness is shown in Fig. 7 and Fig. 8.

Generally, good periodicity of the contours in the spanwise direction can be stated, indicating a regular flow through the cooling pattern. Also, good symmetry in the circumferential direction is obvious around the injection row 1 on the left, confirming the correct adjustment of the showerhead with respect to the main flow direction. The effect of 3D conduction in proximity of the exits is apparent, stemming from internal heat transfer in the cooling holes. The strength of this effect depends on the direction and the exit shape. Since the data analysis is based on 1D heat conduction equation, the data in these zones is not valid, and may therefore not be considered for a quantitative discussion.

The coolant mass flow through the holes varies from one row to another, because of the pressure distribution around the cylinder surface. This can clearly be seen by pronounced streaks of high efficiency behind row 3, which are less distinct or missing at row 2 and 1. It can also be seen that the jet trajectory is sensitive to the blowing ratio: For a low blowing ratio of $G=0.6$, the respective streaks are almost aligned with the main flow direction, whereas they deviate more and more for higher blowing. This is due to the compound angle orientation of the holes and plays an important role for the formation of the cooling film. Good staggering of the jet trajectories results for low coolant injection, yielding good lateral coverage just downstream of the showerhead, whereas the jet trajectories 'merge' for higher blowing. At $G=1.5$, the streaks from row 2 fall right onto the exits of row 3.

For the cylindrical holes at low Mach number, it can be seen that the streaks of rows 2 and 3 are the biggest at $\mathrm{G}=0.6$, and getting smaller as the blowing ratio increases. This effect is known in the literature as jet lift-off, i.e. the coolant gas starts to leave the boundary layer with the increasing momentum of the jet, up to the extreme of an entire loss of the coolant to the main flow, with no cooling effect at all. Along the stagnation line, however, no traces of discrete jets can be distinguished, but rather smeared zones of generally increased effectiveness. The flow situation for injection at stagnation point is considerably different from the other injection stations further downstream. The boundary layer is very thin, main stream velocity is low, and the injection velocity has no component aligned with the main flow. Clearly, the direction of the coolant is highly sensitive to the slightest change of the main flow direction. The effectiveness contours indicate a rather homogeneous distribution of the coolant, and it is proposed that rather than forming distinct jets with defined zones of film coverage, the coolant is injected through the boundary layer, mixes well with the main fluid and then falls back onto the model surface. Only for cases with very high injection mass flow is the coolant lost into the mainstream. At a low blowing ratio of $\mathrm{G}=0.6$, the spanwise averaged effectiveness is gradually building up from relatively low values of 0.2 at the leading edge to a maximum of 0.5 at $45^{\circ}$, and subsequently running out to a level of 0.4 towards higher angles. Local maxima can be seen behind each cooling row. For the higher blowing ratios, the curve is much more flat, on an overall level of about 0.3 . 


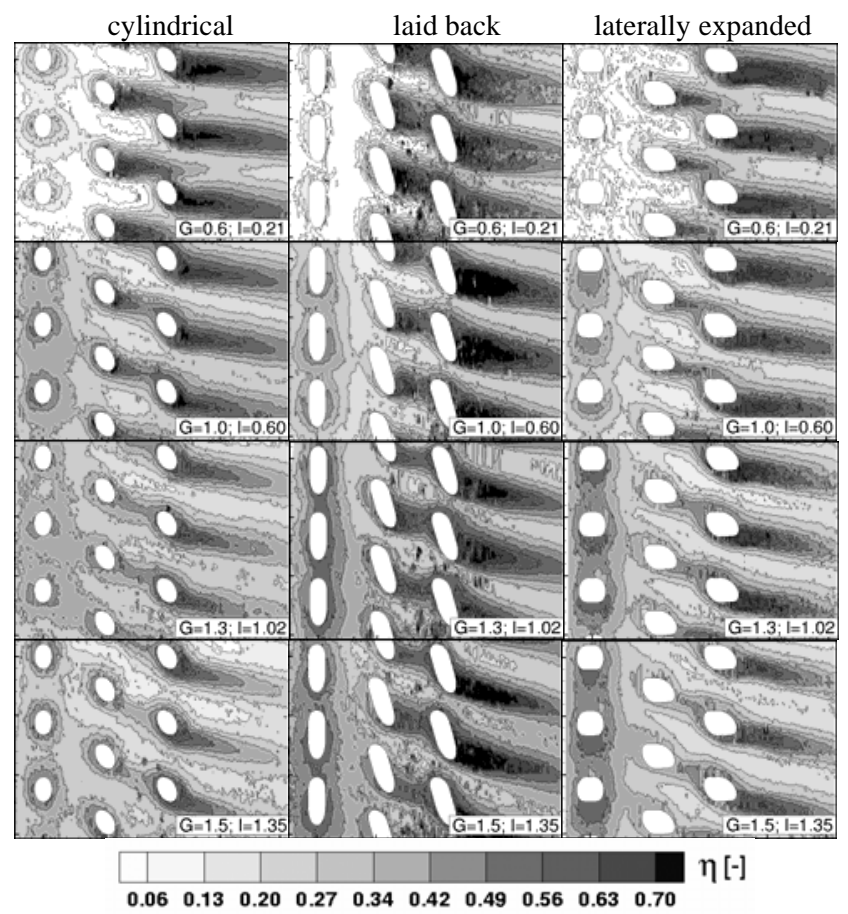

Fig. 5 - Detailed Film Cooling Effectiveness at Low Mach Number $\left(M=0.14 ; R_{D}=8.6 e 4\right)$

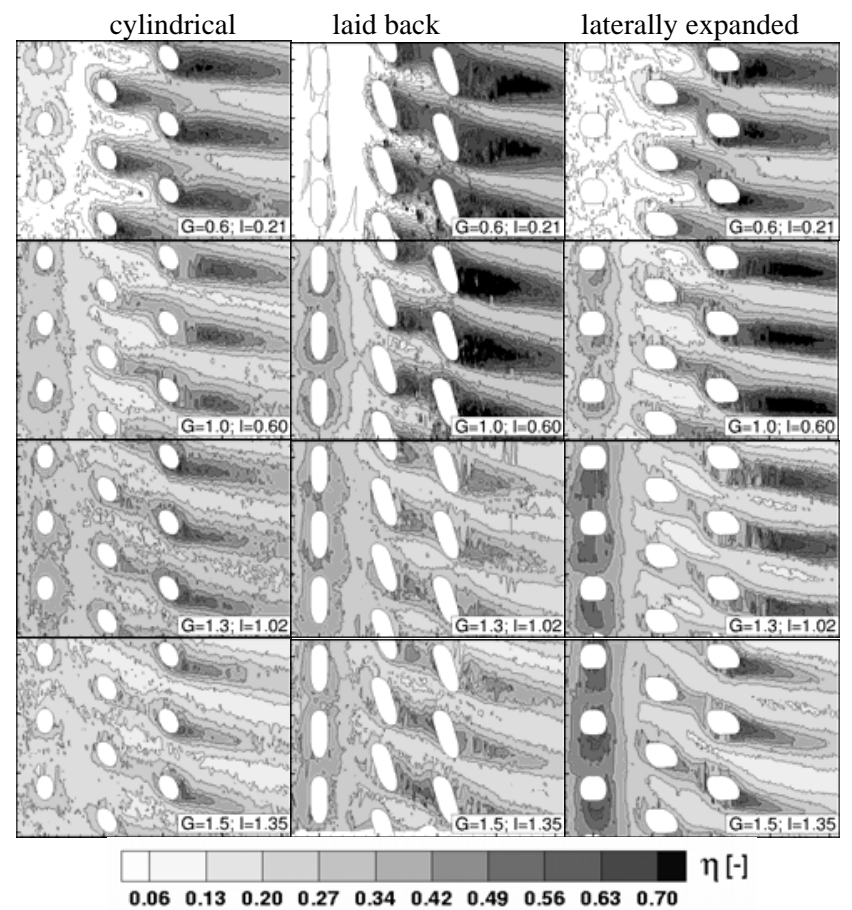

Fig. 6 - Detailed Film Cooling Effectiveness at High Mach Number $\left(M=0.26 ; R_{D}=1.55 e 5\right)$

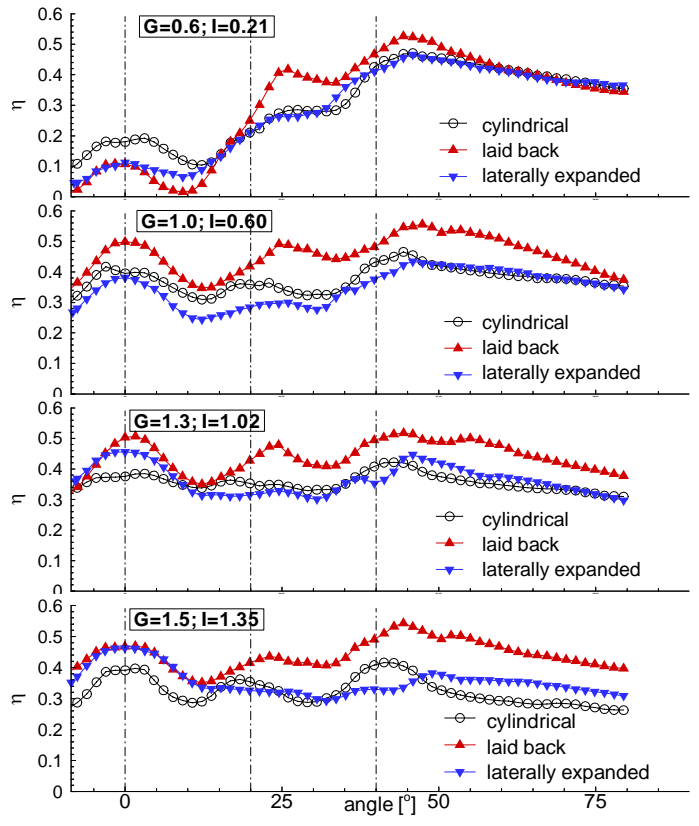

Fig. 7 - Spanwise Averaged Film Cooling Effectiveness at Low Mach Number $\left(M=0.14 ; R_{D}=8.6 e 4\right)$

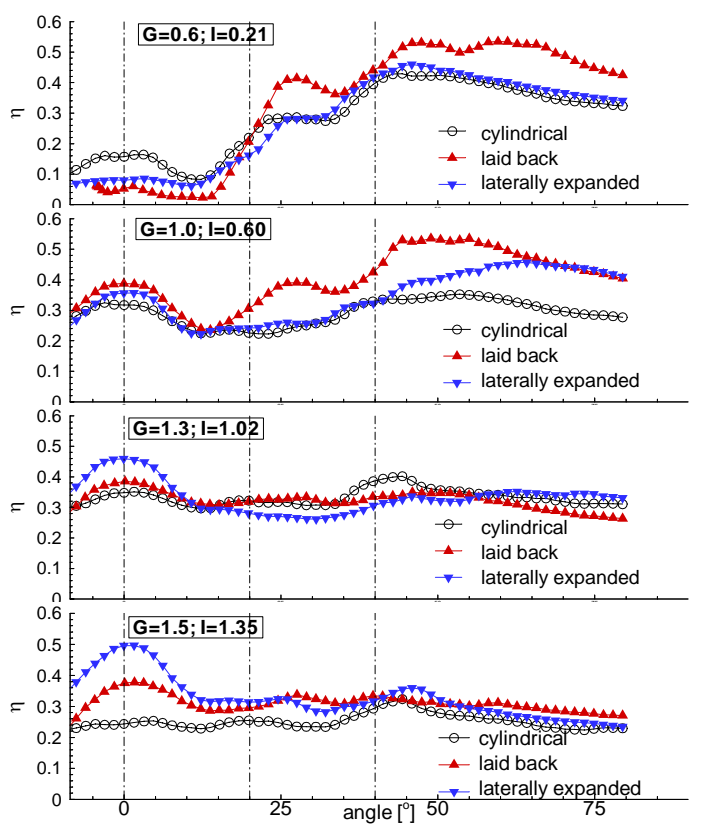

Fig. 8 - Spanwise Averaged Film Cooling Effectiveness at High Mach Number $\left(M=0.26 ; R_{D}=1.55 e 5\right)$ 




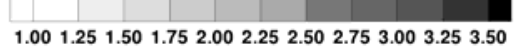

Fig. 9 - Detailed Heat Transfer at Low Mach Number (M=0.14 ; $\operatorname{Re}_{D}=8.6 e 4$ )

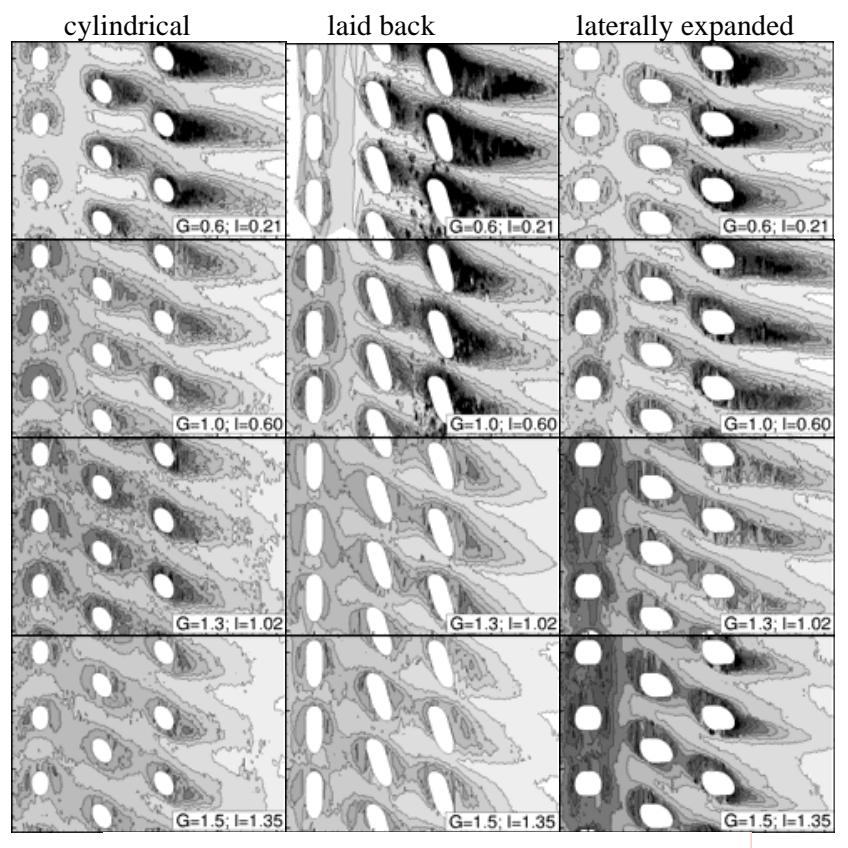

$1.001 .251 .501 .752 .002 .252 .502 .753 .003 .25 \quad 3.50$

Fig. 10 - Detailed Heat Transfer at High Mach Number (M=0.26; $\boldsymbol{R e}_{D}=1.55 e 5$ )

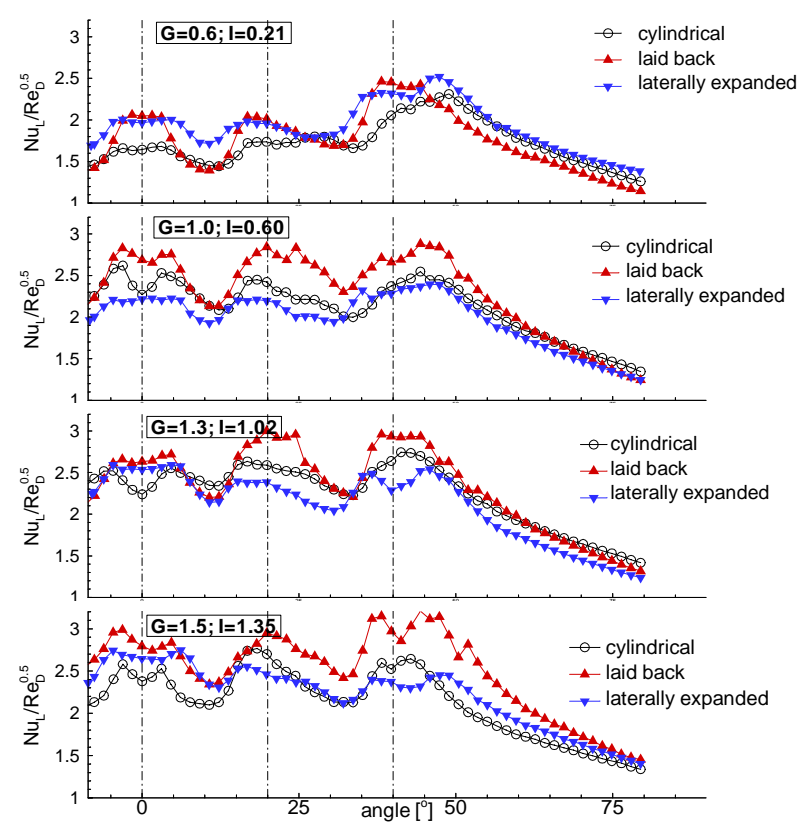

Fig. 11 - Spanwise Averaged Heat Transfer at Low Mach Number $\left(M=0.14 ; R_{D}=8.6 e 4\right)$

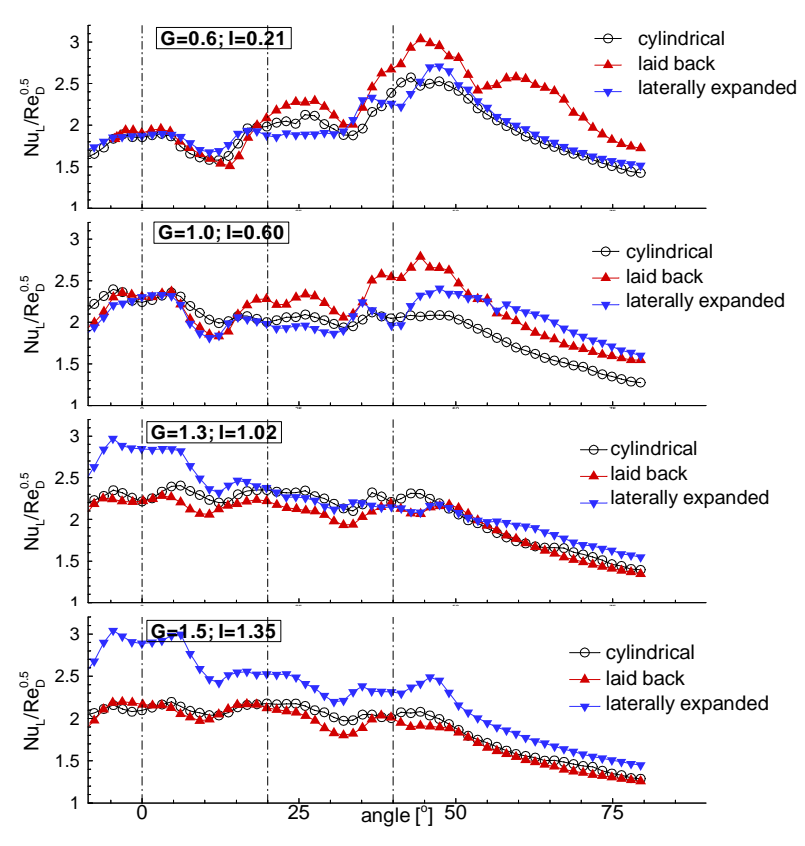

Fig. 12 - Spanwise Averaged Heat Transfer at High Mach Number ( $\left.M=0.26 ; \operatorname{Re}_{D}=1.55 e 5\right)$ 
Laid back holes exhibit generally much broader streaks than cylindrical holes, i.e. better coverage of the surface with the coolant film. Except for the stagnation region, the spanwise averaged effectiveness is higher as indicated in Fig. 7 , giving peak values as high as 0.5 behind the third cooling row.

The laterally expanded holes show effectiveness values on approximately the same level as the cylindrical holes. For higher blowing, they become clearly superior, and the streaks of the third cooling row diminish less rapidly than for the cylindrical holes. This indicates that the jet lift-off is delayed for the laterally expanded holes.

Another phenomenon which is opposed to the described jet lift-off can be seen in the contour plots in Fig. 5 and Fig. 6 : The jets of rows 3 are well attached to the surface at low blowing; then coverage behind the holes improves as coolant gas injection increases, and for further increased blowing, the jets start to detach with subsequent reattachment a little further downstream. This 'drift' of the point of maximum coolant efficiency can most clearly be seen for the laterally expanded holes at low Mach number in Fig. 5. One would expect complete jet detachment at even higher blowing ratios than 1.5. For the cylindrical holes at high Mach number, and even more pronounced for the laterally expanded holes at high Mach number (Fig. 6), an inversion of the tendency can be stated for high blowing: at low blowing ratios they start out with the above described behavior (beginning jet detachment and re-attachment), but for higher blowing ratios, a sudden shift of the maximum of the streaks back to the hole occurs, combined with a further decrease in the level of effectiveness further downstream. The following explanation is proposed: This 'inversion' of the tendency of the jet to detach occurs at high blowing ratios, when the jet trajectories become highly curved, i.e. the jets of the row 2 impinge right onto the jets of row 3 . This may have the effect of bending down the cooling jets of row 3 towards the surface, yielding a jet inclination that is stronger than what would correspond to the hole geometry and injection velocity. Since the coolant still has high momentum a good portion of it leaves the boundary layer a little further downstream. The results are a relatively high effectiveness directly behind the holes, but subsequent loss of the coolant to the main flow. The streaks start directly behind the exit openings and are very short.

A similar effect of favorable interaction between cooling rows has also been reported by Drost and Bölcs (1998), who investigated a double row injection arrangement on the suction side of a turbine blade. The onset of the inversion effect depends on the main flow conditions and the hole geometry. It occurs at $G=1.3$ for the cylindrical holes, and at $\mathrm{G}=1.5$ for laterally expanded holes.

\section{Effect of Mach number}

The measurements indicate that film cooling effectiveness is slightly lower for the high Mach number case. In general, the streaks of high effectiveness behind the exits in Fig. 6 are smaller than in Fig. 5, and the onset of flow separation occurs earlier (i.e. at smaller blowing ratios). The following explanation is proposed: Even though - at constant momentum flux ratio between free stream and coolant higher momentum flow has the tendency of stronger jet deflection due to higher main fluid momentum close to the wall (an effect reported by Mehendale and Han (1993)), a smaller quantity of coolant remains in the boundary layer, since the latter is considerably thinner. Despite the fact that the jet trajectory may be slightly flatter at higher flow conditions, the jet penetration relative to the boundary layer thickness is stronger, and a bigger portion of the coolant gas is lost to the free stream. This explanation is applicable as long as direct 'injection' into the boundary layer is the dominant mechanism for the presence of coolant gas close to the wall, rather than lateral diffusion of coolant from a jet that is only traversing the boundary layer. Which mechanism is predominant may be completely different from one injection geometry to another. All cooling configurations used in this study have injection which is partly aligned with the flow direction due to the hole orientation, i.e. 'direct' injection may play the most important role, and consequently the film effectiveness decreases with increasing Mach number.

\section{Local Heat Transfer}

Detailed surface distributions of non-dimensionalized heat transfer $\mathrm{Nu}_{\mathrm{D}} / \mathrm{Re}_{\mathrm{D}}{ }^{0.5}$ are given in Fig. 9 for the low Mach number case, and in Fig. 10 for the high Mach number case. The corresponding spanwise averaged data are shown in Fig. 11 and Fig. 12. It is apparent in the contour plots that zones of high cooling efficiency behind the holes also have high local heat transfer coefficients. Peak values of up to 3.5 occur for some cases. Around the hole exits, zones of increased heat transfer correspond to regions that are affected by 3D conduction from the holes, as described before in the discussion of cooling effectiveness results. Looking at the spanwise averaged $\mathrm{Nu}_{\mathrm{D}} / \mathrm{Re}_{\mathrm{D}}{ }^{0.5}$ in Fig. 11 or Fig. 12, the overall level of the curves for angles up to $45^{\circ}$ is about $\mathrm{Nu}_{\mathrm{D}} / \mathrm{Re}_{\mathrm{D}}{ }^{0.5}=2-2.5$, with strong fluctuations around the injection openings. A detailed quantitative discussion of spanwise averaged heat transfer results within the showerhead (i.e. surface angles $<45^{\circ}$ ) is not done here, since interpretation of the small differences is difficult, in particular due to the high uncertainty in the near hole regions. Downstream of the actual showerhead $\left(\gamma>45^{\circ}\right)$ the differences between the three cooling configurations are rather small at low Mach number, compared to pronounced differences in cooling effectiveness. Only for the $\mathrm{G}=1.5$ the heat transfer is clearly staggered, being the highest for laid back holes, followed by laterally expanded and cylindrical holes. Maximum values of $\mathrm{Nu}_{\mathrm{D}} / \mathrm{Re}_{\mathrm{D}}{ }^{0.5}=2.5-$ 2.8 occur at $50^{\circ}$, running out to very similar values of $\mathrm{Nu}_{\mathrm{D}} / \mathrm{Re}_{\mathrm{D}}{ }^{0.5}=1$ at $\gamma=80^{\circ}$. At high Mach number, distinct differences in heat transfer occur at $\mathrm{G}=0.6$ and 1.0 , but less pronounced at $\mathrm{G}=1.3$ and $\mathrm{G}=1.5$. Generally, laterally expanded and laid back holes show higher values.

\section{Cooling Performance}

An evaluation of the cooling performance cannot be done solely on the basis of the cooling effectiveness, or the heat transfer, since film cooling involves two adverse effects: reduced effective fluid temperature at the wall on one hand, but also strong mixing and associated increased local heat transfer on the other hand. Therefore, the cooling configurations are compared in terms of the local heat flux ratio $\mathrm{Q}_{\mathrm{f}} / \mathrm{Q}_{\mathrm{o}}$, which relates the local heat flux of the cooled model with an uncooled case, which allows the evaluation of the actual benefit of the film cooling, according to Mehendale et al. (1994)

$$
\frac{Q_{f}}{Q_{0}}=\frac{\alpha_{f}}{\alpha_{0}}\left(1-\frac{\eta}{\Phi}\right)
$$

with the overall cooling effectiveness $\phi$ defined as

$$
\Phi=\frac{T_{w}-T_{g}}{T_{c}-T_{g}}=0.5-0.7
$$

In reality, $\phi$ varies over the surface, depending on the main flow conditions, cooling parameters etc. and this distribution is not known. For this study, one representative value of $\phi=0.6$ was chosen in order to have an indicator of cooling performance. Fig. 13 shows the spanwise averaged heat flux ratio $\mathrm{Q}_{\mathrm{f}} / \mathrm{Q}_{0}$ at four discrete angular positions $10^{\circ}, 30^{\circ}, 50^{\circ}$ and $70^{\circ}$. The first three locations were chosen to be far enough from the hole exits to avoid 3D conduction effects, and close enough to a respective cooling row to show the influence of only this cooling station, aiming to discuss separately the individual cooling rows. The graphs on the left hand side contain results for the low Mach number case, and on the right hand side for the high Mach number case. The last position at $\gamma=70^{\circ}$ is far downstream of the showerhead. For completeness, Fig. 14 contains the corresponding spanwise average effectiveness. 

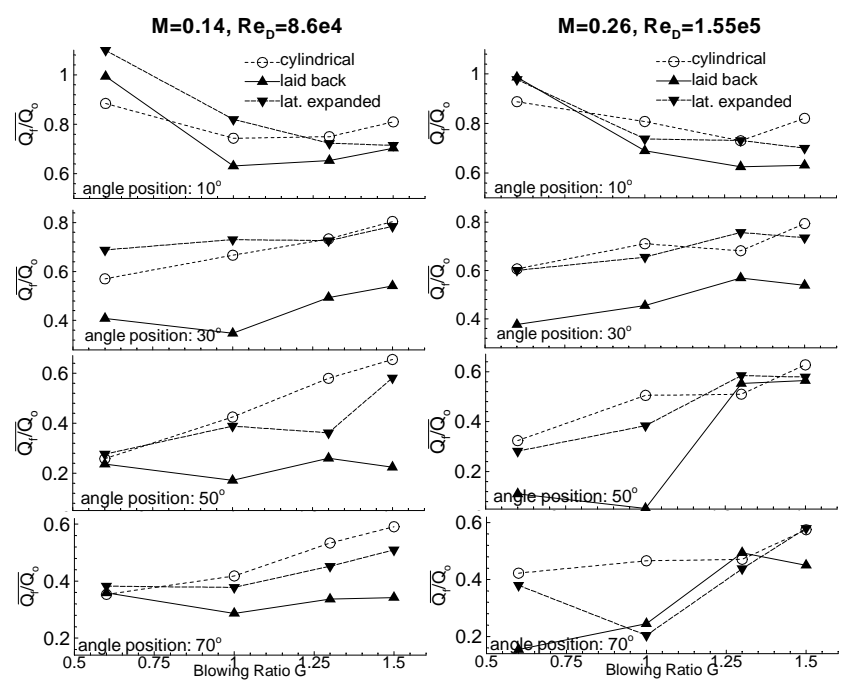

Fig. 13 - Comparison of Cooling Schemes - Spanwise Averaged Heat Flux Ratio at Discrete Surface Positions

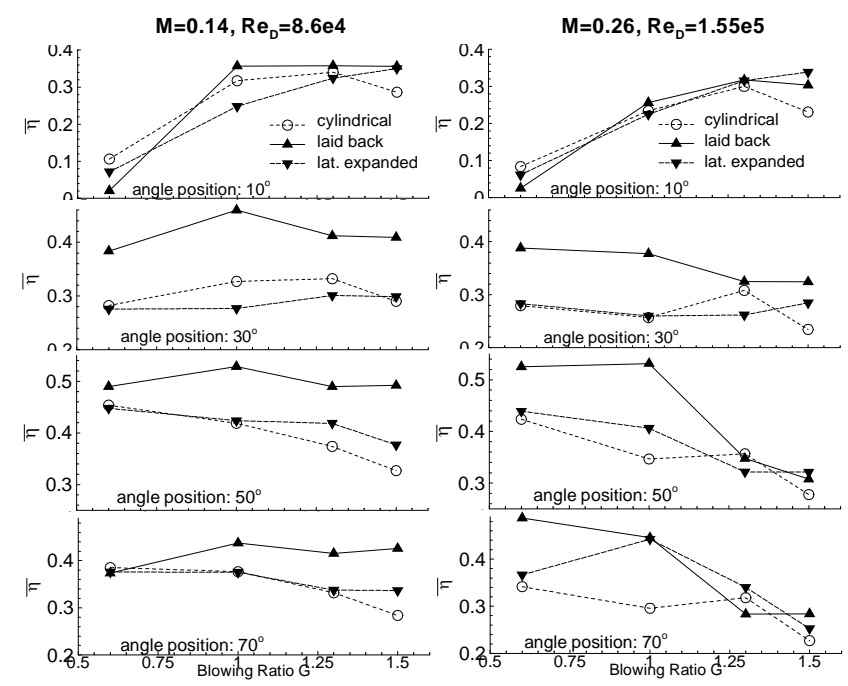

Fig. 14 - Comparison of Cooling Schemes - Spanwise Averaged Film Cooling Effectiveness at Discrete Surface Positions

It is observed that at $\gamma=10^{\circ}$, the heat flux can be reduced by the greatest amount for intermediate blowing ratio with cylindrical and laid back holes, whereas a continuous decrease of the heat flux ratio is seen for laterally expanded holes. The laid back holes at $\mathrm{G}=1.0$ perform best where the heat flux is actually reduced to $65 \%$ with respect to the uncooled case.

Behind row 2, at $\gamma=30^{\circ}$, laid back holes perform clearly better, with a minimum value of down to 0.35 at $G=1.0$, compared to 0.55 or 0.7 for cylindrical and laterally expanded holes, which occur at low blowing values of $\mathrm{G}=0.6$.

Behind row 4, at $\gamma=50^{\circ}$, heat flux ratio is similar for all three hole types at $\mathrm{G}=0.6$, but cooling performance gets worse when increasing $\mathrm{G}$ for cylindrical and laterally expanded holes. Only the laid back type stays at very low levels of $\mathrm{Q}_{\mathrm{f}} / \mathrm{Q}_{0}=0.2$.

Far downstream of the showerhead, at $\gamma=70^{\circ}$, the behavior is comparable to the $50^{\circ}$ station, but with more regularly staggered heat flux ratio when increasing coolant injection.

Generally, there are two adverse phenomena that are apparent:

1. Higher cooling effectiveness associated with reduced heat flux, as can be seen at an angular position of $10^{\circ}$ (plots on top of Fig. 13 and Fig. 14). This may be explained with the presence of more coolant in proximity of the wall, without introducing excessive additional mixing, hence only little rise of the heat transfer coefficient. In that case, the effect of the reduced fluid temperature is predominant and the heat flux is lower.

2. High cooling effectiveness combined with stagnating or even rising heat flux, as it is the case at an angular position of $50^{\circ}$ (third line of plots in Fig. 13 and Fig. 14). Here, the introduction of more coolant into the boundary layer seems to cause considerable additional mixing, associated with strongly increased heat transfer coefficients. In that case, the mixing effect is dominant to the point that it outweighs the favorable effect of the lower fluid temperature at the wall. This results in increased heat flux.

Which one of these two effects is dominant depends on the local injection situation.

\section{CONCLUSIONS}

Heat transfer and film cooling experiments were performed on a cylinder model comparing three cooling configurations in showerhead arrangement, at two free stream flow conditions, $\mathrm{M}=0.14$ and 0.26 , at an elevated turbulence intensity of $\mathrm{Tu}=7 \%$. The cooling configurations consist of five-row arrangements of cylindrical holes with both streamwise compound angle and orientation, and three different exit shapes.

Detailed film cooling efficiency and local heat transfer were presented, and the performance of the cooling schemes was compared in terms of heat load ratio with respect to an uncooled cylinder. The complex character of the formation of cooling films was shown, which is essentially governed by the jet lift-off tendency at high coolant injection rates at downstream positions, whereas right at the stagnation point film formation functions very differently. For some cases, an effect of attachment of jets by upstream cooling rows was found, illustrating the importance of the interaction of individual cooling rows for showerhead cooling.

It was found that holes with 'laid back' type widened exits clearly enhance the overall cooling performance of the showerhead, compared to 'classical cylindrical holes. This was primarily associated with better lateral spread of the individual cooling jets, and to a considerably reduced tendency of jet detachment at higher blowing due to the diffuser effect. Laterally expanded holes performed better than cylindrical holes, but not as well as laid back shaped holes.

\section{ACKNOWLEDGEMENTS}

This study was done within the Brite/EuRam $4^{\text {th }}$ framework, and was funded by the Swiss government. The authors would like to thank all partners of the Brite/Euram $4^{\text {th }}$ framework for their permission for publication.

\section{REFERENCES}

Beeck, A., Fottner, L., Benz, E. and Wittig, S. (1993): The aerodynamic effect of coolant ejection in the leading edge region of a film cooled turbine blade. Heat Transfer and Cooling in Gas Turbines, Vol.AGARD-CP-527, paper 35

Bittlinger, G., Schulz, A. and Wittig, S. (1994): Film cooling effectiveness and heat transfer coefficients for slot injection at high blowing ratios. ASME, IGTA, The Hague, Netherlands, paper 94-GT182 
Drost, U. and Bölcs, A. (1998): Investigation of Detailed Film Cooling Effectiveness and Heat Transfer Distributions on a Gas Turbine Airfoil. ASME, IGTA, Stockholm, Sweden, paper 98-GT-20

Drost, U., Bölcs, A. and Hoffs, A. (1997): Utilization of the Transient Liquid Crystal Technique for Film Cooling Effectiveness and Heat Transfer Investigations on a Flat Plat and a Turbine Airfoil. ASME, IGTA, Orlando, Florida, paper 97-GT-26

Giebert, D., Gritsch, M., Schulz, A. and Wittig, S. (1997): FilmCooling Holes with Expanded Exits: A Comparison of Computational Results with Experiments. ASME, IGTA, Orlando, Florida, paper 97GT-163

Goldstein, R.J., Eckert, E.R.G. and F., B. (1973): Effects of hole geometry and density on three-dimensional film cooling. Journal of Heat and Mass Transfer, Vol.17, pp 595-607

Gritsch, M., Schulz, A. and Wittig, S. (1997): Adiabatic Wall Effectiveness Measurements of Film-Cooling Holes with Expanded Exits. ASME, IGTA, Orlando, Florida, paper 97-GT-164

Hoffs, A., Drost, U. and Bölcs, A. (1997): An Investigation of Effectiveness and Heat Transfer on a Showerhead-Cooled Cylinder. ASME, IGTA, Orlando, Florida, paper 97-GT-69

Mehendale, A.B. and Han, J.-C. (1993): Reynolds number effect on leading edge film effectiveness and heat transfer coefficient. Int. Journal of Heat and Mass Transfer, Vol. 36, 15, pp 3723-3730

Mehendale, A.B., Ou, S., Han, J.-C. and Lee, C.P. (1994): Unsteady Wake over linear turbine blade cascade with air and CO2 film injection: Part II - Effect on film effectiveness and heat transfer distributions. Journal of Turbomachinery, Vol.116, pp 730-737

Vedula, R.J. and Metzger, D.E. (1991): A method for the simultaneous determination of local effectiveness and heat transfer distributions in three-temperature convection situations. ASME, IGTA, Orlando, Florida, paper 91-GT-345

Wittig, S., Schulz, A., Gritsch, M. and Thole, K.A. (1996): Transonic Film-Cooling Investigations: the Effects of Hole Shapes and Orientations. ASME, IGTA, Birmingham, UK, paper 96-GT-222 